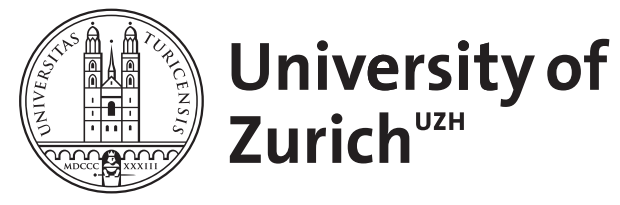

Zurich Open Repository and Archive

University of Zurich

University Library

Strickhofstrasse 39

CH-8057 Zurich

www.zora.uzh.ch

Year: 2016

Der Zusammenhang von Urbanität und Literatur bei Dante

Klinkert, Thomas

DOI: https://doi.org/10.1515/dante-2016-0004

Posted at the Zurich Open Repository and Archive, University of Zurich

ZORA URL: https://doi.org/10.5167/uzh-133749

Journal Article

Published Version

Originally published at:

Klinkert, Thomas (2016). Der Zusammenhang von Urbanität und Literatur bei Dante. Deutsches DanteJahrbuch, 91:23-42.

DOI: https://doi.org/10.1515/dante-2016-0004 


\section{Der Zusammenhang von Urbanität und Literatur bei Dante}

Riassunto: Lo scopo di questo contributo è di analizzare il rapporto tra urbanità e letteratura sulla base dell'esempio di Dante. Il punto di partenza dell'argomentazione è il celebre affresco di Domenico di Michelino (1465), che raffigura la Commedia quale atto di comunicazione rivolto a Firenze, suo destinatario privilegiato. Quest'interpretazione del pittore tocca il cuore dell'opera dantesca, come si cerca di mostrare tramite l'analisi di due canti (Inf. VI e Inf. X). Risulta da quest'analisi che la Commedia può essere considerata il correlato testuale di un ordine del sapere che nasce nell'ambito dello sviluppo sociale, culturale e politico della città di Firenze dei secoli XIII e XIV. All'interno della Commedia si manifestano delle contraddizioni fondamentali. Da una parte, infatti, Dante critica esplicitamente e radicalmente la mentalità affaristica dei fiorentini e le loro discordie politiche; ma dall'altra parte egli è consapevole di dover molto al milieu innovativo di questa città in quanto poeta e pensatore filosofico. Inoltre si cerca di mostrare che il testo dantesco in parte presuppone un lettore che conosca bene dall'interno la situazione di Firenze. Così la dimensione urbana è integrata nella Commedia non solo a livello tematico, ma soprattutto a livello strutturale.

\section{Michelinos Fresko als Ausgangspunkt der Untersuchung von Dantes Verhältnis zu Florenz}

Im Dom von Florenz befindet sich ein allegorisches Gemälde des Malers Domenico di Michelino aus dem Jahr 1465 (Abb. 1). ${ }^{1}$ Auf diesem Bild steht in zentraler,

1 Zur Geschichte und Deutung dieses Bildes vgl. Rudolph Altrocchi, »Michelino’s Dante«, in: Speculum 6 (1931), S. 15-59; Erich Loos, »Das Bild als Deutung von Dichtung. Zur Darstellung Dantes von Domenico di Michelino in Santa Maria del Fiore, Florenz«, in: Lucius Grisebach/ Konrad Renger (Hrsg.), Festschrift für Otto von Simson zum 65. Geburtstag, Frankfurt am Main 1977, S. 160-172; Claudia di Fonzo, »Dante e l'interpretazione iconografica di Domenico di Michelino«, in: Dante 3 (2006), S. 107-114; Elisa Brilli, »Image et autorité au Bas Moyen Âge: l'Allegoria della Commedia par Domenico di Michelino (1465)«, in: Alain Dierkens/Gil Bartholeyns/Thomas Golsenne (Hrsg.), La performance des images, Bruxelles 2010, S. 111-121. - In den vorliegenden Beitrag sind einige Anregungen eingeflossen, die ich von Alice Malzacher und 
vom Betrachter aus gesehen leicht nach rechts versetzter Position der Dichter Dante Alighieri und hält in seiner linken Hand eine aufgeschlagene, von seinem Körper abgewendete Handschrift der Commedia. Mit seiner rechten Hand deutet er zu einer Prozession von Verdammten, die, begleitet von Teufeln, in die Hölle hinabsteigen. ${ }^{2}$ Die Hölle öffnet ihren Schlund in Form einer Felsspalte, und ihr ist in Richtung Bildmitte eine Mauer mit einem Tor vorgelagert. Im Hintergrund des Bildes sieht man den Läuterungsberg, auf dessen Kreisen die Seelen der Verstorbenen sich allmählich nach oben bewegen, in Richtung des auf dem Gipfel gelegenen Irdischen Paradieses, in dem Adam und Eva erkennbar sind, die vor dem Sündenfall dort lebten. Oberhalb des Läuterungsberges erstreckt sich das Himmlische Paradies mit den verschiedenen Sphären, auf denen die Sonne, der Mond und die Gestirne verankert sind. Auf der rechten Seite des Bildes, jener Seite, der Dante sein Gesicht zugewendet hat, erblickt man die Stadt Florenz mit ihrer charakteristischen Domkuppel und einigen berühmten Türmen und Gebäuden. ${ }^{3}$ Diese Stadt ist ebenso wie die auf der linken Bildseite befindliche Hölle von einer Mauer begrenzt. Die beiden Mauern korrespondieren miteinander und sind doch auch verschieden hoch und von unterschiedlicher Farbe. Sie markieren also zugleich ein Ähnlichkeits- und ein Differenzverhältnis zwischen Stadt und Hölle, zwischen Diesseits und Jenseits.

Johanna Gropper erhalten habe. Ihnen möchte ich ebenso danken wie Hans W. Hubert, der mich kunsthistorisch beraten hat. Ebenfalls wichtig waren Gespräche mit Burkhard Hasebrink, FrankRutger Hausmann und Maarten J.F.M. Hoenen.

2 Loos, »Das Bild als Deutung von Dichtung (wie Anm. 1), S. 165, weist darauf hin, dass dem Beschauer »bei näherem Betrachten« deutlich werde, »daß es sich nicht nur um Verdammte schlechthin handelt, sondern daß der Maler die Prozession der Lauen hat darstellen wollen, der sanime triste di coloro, Che visser senza infamia e senza lode` [Inf. III, 35-36]«. So hat das zuvor auch schon Altrocchi, »Michelino's Dante« (wie Anm. 1), S. 40-41, gesehen.

3 Die Darstellung von Florenz ist, so Loos, „Das Bild als Deutung von Dichtung (wie Anm. 1), S. 163, in Bezug auf Dante anachronistisch: „Der gewaltige Dom Santa Maria del Fiore ist zu Lebzeiten des Dichters erst begonnen worden, der Campanile von Giotto erst 1334, also nach Dantes Tod. Ähnliches gilt für den Palazzo della Signoria, an dem seit 1299 gebaut wurde; der Turm als Wahrzeichen der städtischen Macht ist erst 1314 vollendet worden, Dante, der Verbannte, hat ihn also nie sehen können. Auch der Campanile der Badia entstand erst nach 1310.« Loos deutet das Bild so, dass sich in dem "prächtigen Stadtbild « das »stolze Selbstbewußtsein« der Stadt ausdrücke (ebd.). Eine ausführliche Analyse der auf dem Gemälde dargestellten Gebäude findet sich bei Altrocchi, »Michelino's Dante« (wie Anm. 1), S. 28-36, der zu folgender Schlussfolgerung kommt: »The artist was, I repeat, endeavoring to condense in one panorama the salient monuments of Florence and, if I am not mistaken, particularly those which could in some way be related to Dante.«(Ebd., S. 35). 
Dante steht der Stadtmauer von Florenz deutlich näher als der Mauer zur Hölle, und er wendet nicht nur seinen Körper hin zu seiner Heimatstadt, sondern auch seine Schrift. Der mit dem Dichterlorbeer gekrönte Autor ${ }^{4}$ hält jener Stadt, in der er im Jahr 1265 - mithin 200 Jahre vor der Entstehung von Michelinos Gemälde - geboren und wo er aufgrund politischer Verwicklungen und Intrigen im Jahr 1302 in Abwesenheit zum Tode verurteilt wurde, weshalb er gezwungen war, fortan im Exil zu leben, sein Werk entgegen, jene Commedia, die er in den ersten beiden Jahrzehnten des 14. Jahrhunderts geschrieben hat. Er hält sein Weltgedicht jener Stadt entgegen, in der er einst selbst als Politiker tätig und in die Konflikte zwischen weißen und schwarzen Guelfen verwickelt war, jener Stadt, aus deren kultureller und ökonomischer Blüte überhaupt erst die Bedingungen hervorgegangen sind, die ein Werk wie die Commedia möglich gemacht haben - wenngleich sie es natürlich nicht erklären können. ${ }^{5}$

4 Brilli, »Image et autorité au Bas Moyen Âge« (wie Anm. 1), S. 115-116, rückt die der historischen Wahrheit nicht entsprechende Darstellung Dantes als eines poeta laureatus in die Nähe von Par. XXV, 1-9, wo der Erzähler der Commedia sich vorstellt, dass er eines Tages als anerkannter Dichter in seine Heimatstadt zurückkehren werde.

5 Dass so gut wie alle Annahmen über das Leben der empirischen Person Dante Alighieri einen prekären Status besitzen, hat Frank-Rutger Hausmann mit gebührendem Nachdruck hervorgehoben: »Fast alles, was wir von Dante wissen, wissen wir von Dante. Plädoyer für einen kritischen Umgang mit Dantes Biographie«, in: Clausdirk Pollner et al. (Hrsg.), Bright is the Ring of Words. Festschrift für Horst Weinstock, Bonn 1996, S. 109-125. Aus diesem Grund empfiehlt es sich, das Individuum Dante in allgemeine Zusammenhänge seiner Zeit einzuordnen. Eine wichtige Rolle spielt hier die Laienbildung im Florenz des 13. Jahrhunderts als Hintergrund für Dantes Bildung; vgl. August Buck, »Die Commedia«, in: Hans Robert Jauß/Erich Köhler (Hrsg.), Grundriß der Romanischen Literaturen des Mittelalters X/1: Die italienische Literatur im Zeitalter Dantes und am Übergang vom Mittelalter zur Renaissance, Bd. 1: Dantes Commedia und die Dante-Rezeption des 14. und 15. Jahrhunderts, Heidelberg 1987, S. 21-165, hier S. 22: »Für [die Laienbildung] ist Florenz paradigmatisch. Dort begann zuerst das Wissen, verstanden als zweckgebundenes Instrument der Daseinsbewältigung, sich von dem transzendenten Bezug zu lösen, durch den es im christlichen Weltbild gerechtfertigt wurde, nämlich durch seine propädeutische Funktion für die Auslegung der Heiligen Schrift. Neben die reductio artium ad theologiam trat eine Ausrichtung der Bildung auf das Handeln in Staat und Wirtschaft. Trägerin dieser praxisbezogenen Bildung war das im politisch-wirtschaftlichen Leben aktive Laientum der bürgerlichen Oberschicht. Da für eine solche Laienbildung die herkömmlichen Kloster- und Domschulen weder ausreichten noch geeignet erschienen, wurden allerorten neue Stadtschulen eingerichtet: Elementarschulen und die von Villani erwähnten Rechenschulen und Lateinschulen, wobei der Unterricht zum größten Teil in den Händen von Laien lag. « Zu Dantes Bildung vgl. auch Charles Davis, »The Florentine Studia and Dante's >Library «, in: Giuseppe di Scipio/Aldo Scaglione (Hrsg.), The Divine Comedy and the Encyclopedia of Arts and Sciences, Amsterdam/Philadelphia 1988, S. 339-366. 


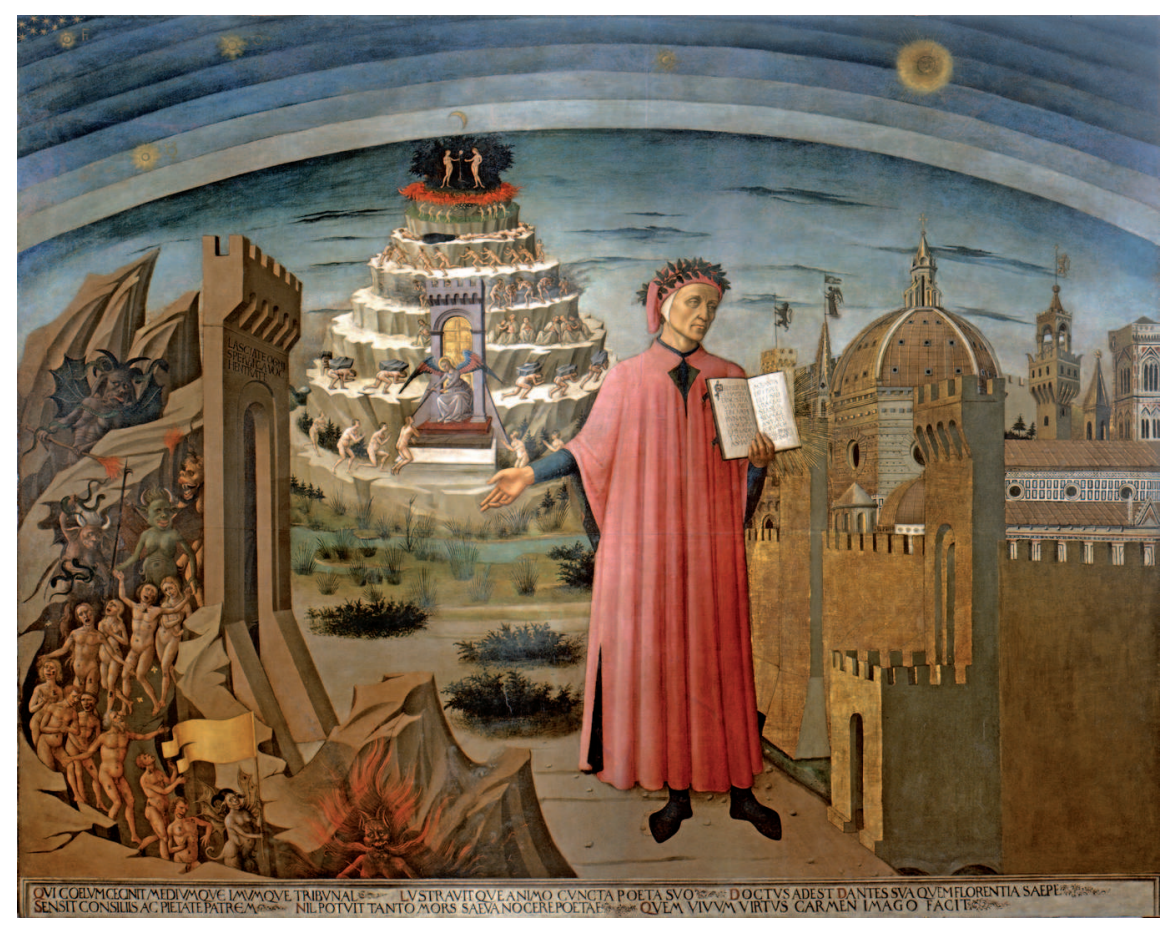

Abb. 1: Domenico di Michelino, Die Allegorie der Göttlichen Komödie (1465)

Dante ist zweifellos der erste nachantike Dichter, der sich in seinen Werken intensiv mit dem Städtischen auseinandersetzt. Während wichtige Gattungen der volkssprachlichen Literatur des Hochmittelalters (höfische Lyrik, Heldenepik, Artusroman) an den Hof als ihr soziales und normatives Zentrum gebunden waren, verschiebt sich bei Dante als dem Erben dieser Tradition der Fokus auf die Stadt. Die von den Provenzalen entwickelte Semantik der höfischen Liebe, welche Dante rezipiert, wird in der Vita nova dezidiert in den Raum der Stadt Florenz eingebettet; so bezeichnet George Holmes die Vita nova trefflich als »a new city literature springing out of an embryonic city culture «. ${ }^{6}$ Auch die Commedia, die mit ihrem universalistischen Anspruch auf das Ganze der Schöpfung und der Seinsordnung zielt, gewinnt ihr spezifisches Profil vor dem Hintergrund der sozialen und politischen Entwicklungen der Stadt Florenz im 13. und frühen 14. Jahrhundert. Diesbezüglich besonders aufschlussreich sind die polemischen Invektiven gegen Florenz und das dort herrschende ökonomische Gewinnstreben

6 George Holmes, Florence, Rome and the Origins of the Renaissance, Oxford 1986, S. 126. 
(die »súbiti guadagni«, Inf. XVI, 73), ${ }^{7}$ welches von Dante in Opposition gesetzt wird $\mathrm{zu}$ einem verlorenen Ideal der Frömmigkeit und Einfachheit, für welches sein Urahn Cacciaguida (Par. XV-XVII) einsteht. Ebenfalls bemerkenswert ist die Tatsache, dass die Topographie der Hölle z. T. auf das Raummodell der Stadt zurückgreift. Indem Dante zahlreiche Bewohner von Florenz (und anderen italienischen Städten) im Jenseits auftreten lässt, erweist er sich, wie schon Erich Auerbach gezeigt hat, als »Dichter der irdischen Welt «. ${ }^{8}$

Was sich auf politischer Ebene als antagonistisches Verhältnis artikuliert, lässt sich auf epistemologischer Ebene als komplexe und spannungsreiche Wechselwirkung beschreiben. Denn so sehr sich Dante einerseits mit der Commedia in Gegnerschaft zu seiner Heimatstadt stellt, so wenig wäre dieses Werk andererseits denkbar ohne die Bildungsvoraussetzungen, welche in Florenz im Zuge der ökonomischen Entwicklung des 13. Jahrhunderts geschaffen wurden und von denen der Autor profitieren konnte. ${ }^{9}$ Dank dieser Bildung, die er vermutlich im Exil noch vertiefen und erweitern konnte, kann Dante in seinem Hauptwerk das gesamte (politische, geographische, theologische, philosophische usw.) Wissen seiner Zeit bündeln; ${ }^{10}$ und er kann mit einem gebildeten städtischen Laienpublikum rechnen, das dieser Wissensbündelung etwas abzugewinnen vermag. Indem der Jenseitswanderer >Dante als Protagonist der Commedia in die letzten Geheimnisse der Schöpfung eingeweiht wird, entwirft der Text zugleich einen impliziten, seinem Habitus nach städtischen Leser, der im Nachvollzug von >Dantes` Initiati-

7 Die Commedia wird nach folgender Ausgabe zitiert: Dante Alighieri, La Commedia secondo l'Antica Vulgata. Testo critico stabilito da Giorgio Petrocchi per l'edizione nazionale della Società Dantesca Italiana, Torino 1975.

8 Erich Auerbach, Dante als Dichter der irdischen Welt, Berlin/Leipzig 1929. Zur zentralen Bedeutung von Florenz für die Commedia vgl. Eugenio Ragni, »F[irenze] nell'opera dantesca«, in: Umberto Bosco (Hrsg.), Enciclopedia dantesca, Bd. II, Roma 1970, S. 920-927, hier S. 920: »Nessun'altra città ha mai assunto in un'opera d'arte un ruolo di così intensa e viva presenza, nessun'altra è mai stata caricata del peso di un'esperienza passionale altrettanto drammatica quanto F[irenze], indubbia deuteragonista della Commedia e, come tale, luogo dei punti di ogni tensione sentimentale del suo grande cittadino; il quale, exul inmeritus, privato della presenza ,fisica della città, ad essa - alle sue istituzioni, alle sue miserie, ai suoi splendori, alle sue colpe sembra voler rapportare ogni altra realtà, storica ideale o morale, manifestando in questo rapporto tutta la carica di una mentalità tipicamente medievale, sensualmente avvinta al microcosmo della città comunale [...], assumendo nel contempo quello stesso microcosmo a fondamenta del mirabile edificio della sua opera [...].«

9 Charles T. Davis, "Education in Dante's Florence«, in: ders. (Hrsg.), Dante’s Italy. And other Essays, Philadelphia 1984, S. 137-165; August Buck, »Die Commedia« (wie Anm. 5), S. 21-29.

$10 \mathrm{Zu}$ den Quellen des von Dante verarbeiteten Wissens vgl. Frank-Rutger Hausmann, »Dantes Kosmographie - Jerusalem als Nabel der Welt«, in: Deutsches Dante-Jahrbuch 63 (1988), S. 7-46. 
onsreise dieses Wissens ebenfalls teilhaftig wird. Insofern ist dieses Werk auf einer basalen Ebene ein Indikator des Wissens seiner Epoche. ${ }^{11}$

Indem der Text auf der Grundlage dieses Wissens ein hochkomplexes und singuläres poetisches Weltmodell entwirft, überschreitet die Commedia jedoch die Wissensordnung ihrer Zeit. Dieses auf der Ebene der "sekundären Modellbildung « ${ }^{12}$ sich manifestierende transgressive Moment der Divina Commedia korreliert mit der Tatsache, dass dieser Text eine vor dem Hintergrund mittelalterlicher Wissensmodelle höchst problematische diskursive Anomalie darstellt. Denn es handelt sich um eine poetische Fiktion, welche für sich in Anspruch nimmt, nach den Interpretationsregeln des eigentlich für die Heilige Schrift reservierten mehrfachen Schriftsinns gelesen zu werden - so jedenfalls wird es in der Dante zugeschriebenen Epistel an Cangrande della Scala postuliert. Gerhard Regn spricht treffend von Dantes "paradoxem Unterfangen«, welches gerichtet sei auf »die dichterische Beglaubigung der Anagogie durch eine Erzählung, die im Zuge ihrer Entfaltung die Differenz zwischen Fiktion und Wirklichkeit zugleich setzt und kassiert «. ${ }^{13}$ Die aporetische Struktur der Commedia lässt sich auf der Ebene des Textes selbst nachweisen, etwa wenn >Dante Schattenleib des Florentiners Ciacco begegnet und dieser ihn bittet, nach seiner Rückkehr ins Diesseits von seiner Begegnung mit ihm Zeugnis abzulegen. Der damit ausgedrückte Wunsch, an die Taten des Verstorbenen zu erinnern, steht zwar im Einklang mit der der Dichtung seit der Antike gestellten Aufgabe, am kulturellen Gedächtnis mitzuwirken; andererseits entsteht jedoch ein Widerspruch zwischen der Dichtung und der göttlichen Jenseitsordnung, die im Falle Ciaccos durch die Unkenntlichmachung von dessen Schattenleib das Gedächtnis an den Sünder im Zuge einer damnatio memoriae löscht. Spannungen und Widersprüche lassen sich auch auf intertextueller Ebene zeigen, etwa wenn Dante bestimmte Theorien und Wissensmodelle seiner Zeit aufgreift und poetisch umdeutet. $^{14}$

11 Vincenzo Vita, »L'unità del sapere nella Divina Commedia«, in: Esperienze Letterarie 9, 2 (1984), S. 103-118.

12 Jurij M. Lotman, Die Struktur literarischer Texte, übers. v. Rolf-Dietrich Keil, München 1972, S. $22 \mathrm{ff}$.

13 Gerhard Regn, »Gott als Dichter: Die Wirklichkeit der Fiktion in Dantes Paradiso«, in: Ursula Peters/Rainer Warning (Hrsg.), Fiktion und Fiktionalität in den Literaturen des Mittelalters, München 2009, S. 365-385, hier S. 366.

14 Vgl. etwa Katharina Münchberg, Dante. Die Möglichkeit der Kunst, Heidelberg 2005, S. 34, die am Beispiel der in der Scholastik theoretisch beschriebenen Kategorie der Potentialität zeigt, dass Dante »die Kluft zwischen der Potentialität des Kunstwerks und der Aktualität des Schönen zu überbrücken [versucht], um die Identität von Potentialität und Aktualität in der Immanenz des Werks zu erreichen«. 
Solche strukturellen Widersprüche korrelieren, so die hier vertretene Auffassung, mit dem widersprüchlichen Verhältnis von Dantes poetischem Hauptwerk zu seiner Heimatstadt Florenz, der sich dieses Werk zugleich verdankt und gegen die es sich richtet. Insofern ist die fundamental widersprüchliche Textstruktur als eine besondere Manifestation von Urbanität zu deuten, gewissermaßen als Inkorporation der durch die Entwicklung der Stadt Florenz gegebenen sozialen, politischen, wirtschaftlichen, religiösen und epistemischen Bedingungen und der damit verbundenen Konflikt- und Konkurrenzsituation in den poetischen Kommunikationsakt.

In der Forschung wurde dies bisher so pointiert noch nicht herausgearbeitet. Zum Verhältnis von Dante und Florenz gibt es Darstellungen wie die von John M. Najemy, ${ }^{15}$ in welcher der Schwerpunkt allerdings auf die politischen und sozialen Verhältnisse gelegt und die These vertreten wird, dass Dantes auf den ersten Blick generelle Kritik an Florenz in Wahrheit eine gegen die »upper class of large, rich, and politically influential families « ${ }^{16}$ gerichtete sei; gegen diese Oberschicht mache Dante sich die Position des popolo zu eigen, von der er sich allerdings dadurch grundlegend unterscheide, dass für ihn als ein autonomes Ganzes nicht die Stadt gelten könne, sondern die gesamte Menschheit, welche historisch unter der Führung des antiken Rom stehe. ${ }^{17}$ Andere Untersuchungen versuchen Dantes Rolle als Florentiner bzw. als Florentiner Politiker zu umreißen, ${ }^{18}$ oder sie widmen sich der Darstellung der Stadt Florenz in der Commedia, etwa in den Cacciaguida-Gesängen des Paradiso. ${ }^{19}$ Die Überblicksdarstellung von Giuliano Tanturli zum Wandel des Florenzbildes in Dantes Werken beschränkt sich weitgehend auf die Nennung einschlägiger Textstellen und ihre Paraphrasierung. ${ }^{20} \mathrm{Zu}$ den brisanten Fragen, welche Dantes Commedia als poetischer Text dem Leser stellt, stoßen solche am Stofflichen orientierte Untersuchungen nicht vor. Für den vorliegenden Zusammenhang relevantere Studien bringen die politi-

15 John M. Najemy, »Dante and Florence«, in: Rachel Jacoff (Hrsg.), The Cambridge Companion to Dante, Cambridge 1993, S. 80-99.

16 Ebd., S. 87.

17 Ebd., S. 96.

18 Peter Herde, „Dante als Florentiner Politiker«, in: ders., Von Dante zum Risorgimento. Gesammelte Abhandlungen und Aufsätze, Bd. I, Stuttgart 1997, S. 1-54; R.W.B. Lewis, „Dante the Florentine«, in: The Yale Review 89, 3 (2001), S. 1-10. Was hierbei allerdings selten angemessen berücksichtigt wird, ist der unsichere Status dessen, was wir über Dantes Leben zu wissen glauben; vgl. hierzu Hausmann, »Fast alles, was wir von Dante wissen, wissen wir von Dante« (wie Anm. 5).

19 Vgl. etwa Jeffrey T. Schnapp, The Transfiguration of History at the Center of Dante's »Paradise«, Princeton 1986, insbes. S. 36-69.

20 Giuliano Tanturli, "L'immagine topografica di Firenze nella poesia di Dante«, in: Michelangelo Picone (Hrsg.), Dante. Da Firenze all'aldilà, Firenze 2001, S. 263-273. 
schen, sozialen und ökonomischen Gegebenheiten im Florenz Dantes in konstitutive Verbindung mit seiner Dichtung. Hier ist etwa Patrizia Mainoni zu nennen, die eine Neulektüre der Commedia unter ökonomiegeschichtlichem Blickwinkel vorschlägt, indem konkrete Textstellen aus dem Themenkreis von Wirtschaft und Finanzwesen in der Commedia und ihren Kommentaren mit den ökonomischen Bedingungen der Zeit in Relation gesetzt werden. ${ }^{21}$ Susan Noakes stellt die These auf, dass die Entwicklung des Bankwesens in der Toskana des Duecento entscheidenden Einfluss auf alle Diskurse ausgeübt habe. Ihr zufolge ist der Markt ein Strukturmodell für die Commedia, und sie schließt mit dem Desiderat, ausgehend von den punktuellen und ausbaufähigen Beobachtungen ihres Aufsatzes die ökonomischen Bezüge der Commedia umfassend aufzuarbeiten und zum kulturellen Kontext in Beziehung $\mathrm{zu}$ setzen. ${ }^{22}$ An diese Studien kann gewinnbringend angeknüpft werden, weil sie nicht am Thematischen (Stadt, Politik, Wirtschaft) hängenbleiben, sondern eine Neulektüre der Commedia aus der Perspektive eines komplexen historischen Beschreibungsmodells vornehmen und den Zusammenhang von historischen Bedingungen und Textstrukturen aufzudecken versuchen. Allerdings soll hier der Fokus nicht auf das Ökonomische, sondern auf das diesem übergeordnete Urbane gelegt werden. ${ }^{23}$

Blicken wir daher noch einmal auf Michelinos Dante-Gemälde. Besonders auffällig ist die schon erwähnte Korrespondenz zwischen der teilweise stadtförmigen, teilweise in sich zusammenbrechenden, felsigen Sphäre der Hölle und der durch Mauerwerk und architektonische Ordnung gekennzeichneten Stadt Florenz. Beide befinden sich zusammen mit Dante im Vordergrund des Bildes, und

21 Patrizia Mainoni, »L'orizzonte economico medievale nella Divina Commedia e nei principali commenti del Trecento«, in: Giuseppe di Scipio/Aldo Scaglione (Hrsg.), The »Divine Comedy « and the Encyclopedia of Arts and Sciences, Amsterdam/Philadelphia 1988, S. 315-338.

22 Susan Noakes, „Dante e lo sviluppo delle istituzioni bancarie a Firenze: ıi sùbiti guadagni «", in: Michelangelo Picone (Hrsg.), Dante. Da Firenze all'aldilà, Firenze 2001, S. 249-261. Vgl. hierzu neuerdings Cornelia Klettke, »Die Abwägung irdischer und himmlischer Güter - ökonomische Ethik und Seelenökonomie in Dantes Commedia«, in: Deutsches Dante-Jahrbuch 87/88 (2012/13), S. 163-202, wo gezeigt wird, dass Dantes Verhältnis zu Gold und Geld ambivalent ist, insofern den Invektiven gegen Geiz, Wucher und Habgier eine »Poetik des Goldes und des Geldes« (S. 166 ff.) gegenübersteht, die »auf einem elaborierten rhetorischen Begriffsrepertoire [beruht], dessen Metaphorik eine Tauschökonomie suggeriert, indem im eigentlichen Sinn konkret vorstellbare irdische Preziosen und Reichtümer durch Permutation in geistlich-seelische Glückseligkeiten transformiert werden« (S. 190). Das kritisierte Tauschmittel Geld wird somit auch, so Klettke, zum metaphorischen Modell der Dante'schen écriture und des von ihr Dargestellten.

23 Vgl. hierzu auch meine Untersuchung »Florenz und die Divina Commedia. Zum Zusammenhang von städtischem Habitus und literarischem Text im italienischen Spätmittelalter «, in: Angelika Corbineau-Hoffmann/Pascal Nicklas (Hrsg.), Die Dynamik der Metropolen - von der Antike bis zur Postmoderne, Berlin/Boston (im Druck). 
Dantes Körper vermittelt gewissermaßen, durch Gestik und Haltung, zwischen seiner Heimatstadt und der Hölle. Darin kann man Dantes politische Situation, als die des außerhalb der Stadt, im Exil sein Werk schreibenden Autors, ebenso erkennen wie den politischen Anspruch dieses Werkes, die insbesondere im ersten und sicherlich meistgelesenen Teil, dem Inferno, sich manifestierende fundamentale Kritik, welche Dante gegen die Bewohner seiner Stadt, denen er Streit, Neid und Habgier vorwarf, gerichtet hat. Man kann ohne Weiteres eine Bilanz aufmachen, die belegt, mit welcher Verve Dante in der Commedia die Bewohner von Florenz als bevorzugte Zielscheibe des göttlichen Strafgerichts hat auftreten lassen. ${ }^{24}$ Seine Schrift kann man in dieser Perspektive als eine Art >Racheakt $\iota$ des Verbannten interpretieren. Der Autor hält den Bewohnern seiner Heimatstadt die im Übrigen auf dem Bild überhaupt nicht $\mathrm{zu}$ sehen sind, denn die Stadt erscheint hier als menschenleere Ansammlung von Gebäuden, während die beiden auf Erden situierten Jenseitsreiche mit menschlichen Körpern bevölkert sind - gewissermaßen den Spiegel vor, um ihnen zu zeigen, was es für Konsequenzen hat, wenn man sich so falsch und sündhaft verhält, wie sie es Dantes Auffassung nach getan haben. ${ }^{25}$

Dantes Werk wird auf dem Bild von Domenico di Michelino verdoppelt. Zum einen sieht man es als materiellen Textkörper, als Signifikanten, zum anderen erblickt man das Signifikat dieses Signifikanten, d. h. die von diesem Text durch Schrift erzeugte Welt in ihren drei Dimensionen, den drei Jenseitsreichen, die allerdings auf dem Bild, entgegen der Raumstruktur, wie sie Dantes Gedicht

24 Man denke an die Florentiner Ciacco (Inf. VI), Filippo Argenti (Inf. VIII), Farinata und Cavalcante (Inf. X), den namenlosen Selbstmörder (Inf. XIII), Brunetto Latini und Andrea de' Mozzi (Inf. XV), Guido Guerra, Tegghiaio Aldobrandi, Iacopo Rusticucci und Guglielmo Borsiere (Inf. XVI), Gianni di Buiamonte de' Becchi (Inf. XVII), die aus Bologna stammenden Florentiner podestà Catalano dei Malavolti und Loderingo degli Andalò (Inf. XXIII), Cianfa Donati und Buoso (Inf. XXV), Mosca dei Lamberti (Inf. XXVIII), Geri del Bello (Inf. XXIX), Gianni Schicchi (Inf. XXX) und Bocca degli Abati (Inf. XXXII).

25 Selbstverständlich wäre bei einer Gesamtdeutung des Bildes, die hier allerdings nicht beabsichtigt ist, zu berücksichtigen, dass es im Auftrag der Signoria und vermutlich des Florentiner Kanzlers Bartolomeo Scala entstand und dass es - neben der Preisung Dantes - vor allem auch eine Selbstpreisung der Stadt zum Ausdruck bringen sollte. Eine differenzierte Interpretation findet sich bei Brilli, »Image et autorité au Bas Moyen Âge« (wie Anm. 1), S. 119, die drei Wirksamkeiten (»trois façons d'être efficace«) des Gemäldes ausmacht: (1) seine Wirksamkeit als Substitut des in Florenz nicht vorhandenen Grabmals des Dichters; (2) seine Wirksamkeit als Simulation von Dantes Rückkehr nach Florenz und seiner Dichterkrönung, und somit als Buße für das schuldhafte Verhalten der Florentiner gegenüber Dante (»comme solde de la faute commise par la ville envers lui«); (3) seine Wirksamkeit als Umkehrung von Dantes polemischer Haltung gegenüber seiner Heimatstadt. Gerade durch die hiermit angesprochene Umkehrung aber wird die Opposition zwischen Dante und Florenz bloßgelegt. 
zugrunde liegt, in einer unmittelbaren Nähe zueinander visualisiert werden. Das Bild ist somit nicht als eine mimetische Darstellung von Dantes Jenseitsreichen zu betrachten, sondern als eine allegorische Verdichtung der in der Commedia beschriebenen Welt und ihres Ausgangs- und Bezugspunktes, der Stadt Florenz. Man kann das Bild so interpretieren, dass der Maler den Autor Dante und die Stadt Florenz in einen Dialog zueinander stellt. Florenz wird auf dem Bild ganz eindeutig als privilegierter Adressat der Commedia markiert. ${ }^{26}$ Zugleich verweist die visuelle Korrespondenz zwischen der Hölle und der Stadt, sowohl was deren Positionierung auf dem Bild, als auch was deren Einrahmung durch Mauerwerke betrifft, auf die Tatsache, dass Dante sein Jenseitsreich zumindest teilweise als eine Art unterirdischer Stadt konzipiert hat, wofür er Begriffe wie »nobile castello« (Inf. IV, 106), »la città c'ha nome Dite« (Inf. VIII, 68) oder »la città del foco« (Inf. X, 22) verwendet. Innerhalb des gigantischen Höllenkraters befinden sich städtische Strukturen, und es begegnen dem Jenseitswanderer >Dante Hölle zahlreiche Stadtbewohner, insbesondere Florentiner. Mit seiner allegorischen Verdichtung trifft der Maler somit einen wesentlichen Aspekt von Dantes Werk. Diesen Aspekt, nämlich den Zusammenhang von Urbanität und Literatur, möchte ich im Folgenden weiter ausführen.

\section{Die paradigmatische Funktion der Begegnung mit Ciacco (Inf. VI)}

Bei seiner Jenseitsreise begegnet >Dante zahlreichen Personen, die aus seiner Heimatstadt Florenz stammen. In den Gesprächen mit diesen Personen wird immer wieder die Geschichte der Stadt - und damit auch die Geschichte 'Dantes` - thematisiert. Der erste Florentiner, dem der Wanderer in der Hölle begegnet, heißt Ciacco (Inf. VI). Er befindet sich im dritten Kreis der Hölle; seine Sünde ist die der Schlemmerei, die durch einen immerwährenden Eisregen bestraft wird. Die zu ewigen Qualen verdammten Sünder liegen auf dem Boden, und als >Dante mit Vergil vorbeikommt, erhebt sich einer von ihnen und spricht ihn an. Er fordert ihn auf, ihn zu erkennen, was >Dante Ciaccos Schattenkörper durch die Höllenqualen zur Unkenntlichkeit entstellt worden ist. Darauf stellt Ciacco sich selbst vor und bettet seine Geschichte in die

26 So sieht dies auch Ragni, »F[irenze] nell'opera dantesca« (wie Anm. 8), S. 921: »[...] i Fiorentini, cui la Commedia era in un certo senso particolarmente destinata sia quale dimostrazione dell'altezza culturale e morale raggiunta dall'exul inmeritus (e perciò prova per lui della sua innocenza), sia come terribile ammonimento di una prossima e terribile punizione.« 
Situation der Stadt Florenz ein. Er formuliert eine deutliche Kritik an den Bürgern seiner Heimatstadt: »La tua città, ch'è piena / d'invidia sí che già trabocca il sacco« (»So voll bösem Neide / Ist deine Heimat, daß der Sack am Bersten. «; Inf. VI, 49-50). ${ }^{27}$ Ciacco und `Dante sind sich hinsichtlich der negativen Beurteilung der Florentiner einig. Zur Charakterisierung verwenden sie Begriffe wie »invidia« (V. 50), »discordia« (V. 63), »superbia« und »avarizia« (V. 74). In einer Prophezeiung erklärt Ciacco schließlich, dass es in Florenz zum blutigen Bürgerkrieg kommen werde. Damit steht dieser Florentiner in einer Reihe mit anderen Figuren der Commedia, die ebenso wie der Autor und Protagonist aus Florenz stammen und diesem die Zukunft vorhersagen (Farinata degli Uberti, Inf. X, Brunetto Latini, Inf. XV, Cacciaguida, Par. XV-XVII). Diese Florentiner nehmen eine kritische Perspektive auf ihre Heimatstadt ein, die der des exilierten Dante nahekommt. Sie werden zu Sprachrohren des Autors. Die drei Schlagworte »superbia«, »invidia« und »avarizia«, die später erneut auftauchen, zum Beispiel in Inf. XV, 68, haben in der Rezeption sprichwörtlichen Charakter angenommen und werden bei Autoren wie Giovanni Villani oder Matteo Frescobaldi aufgegriffen. ${ }^{28}$ Dante inkriminiert mit diesen Vorwürfen die Selbstzerfleischung der florentinischen Parteien, ihr Machtstreben und ihren Hochmut sowie die Dominanz des wirtschaftlichen Gewinnstrebens.

Auffällig ist, dass der Florentiner Ciacco den Florentiner `Dante`, von dessen göttlich legitimierter Jenseitsreise er nichts wissen kann, sofort als Bewohner der gemeinsamen Heimatstadt identifiziert, während umgekehrt >Dante` seinen Landsmann dagegen nicht erkennt. Daraus lässt sich schlussfolgern, dass der Jenseitswanderer als Florentiner an seiner Kleidung und seinem Habitus erkennbar sein muss. Außerdem ist ein für diesen Abschnitt charakteristisches Prinzip der Gegensatz von Schein und Sein. >Dante fragt Ciacco, ob es denn in Florenz noch Gerechte gebe, und nennt einige Florentiner, die er für rechtschaffen hält. Darauf antwortet Ciacco, dass diese - es handelt sich um Farinata degli Uberti, Iacopo Rusticucci und andere - zu den »anime piú nere« (Inf. VI, 85) gehören, die ebenfalls in der Hölle schmoren, und zwar noch weiter unten als er selbst. In Florenz ist offenbar vieles ganz anders, als es auf den ersten Blick zu sein scheint.

Diese erste Begegnung mit einem Florentiner in der Hölle erfüllt verschiedene Funktionen: Sie ermöglicht >Dante einen Austausch mit einem Landsmann, der, ähnlich wie er selbst, der gemeinsamen Heimatstadt äußerst kritisch gegenüber-

27 Ich zitiere nach folgender deutscher Übersetzung: Dante Alighieri, Die Göttliche Komödie. Übersetzt von Hermann Gmelin. Mit Anmerkungen und einem Nachwort von Rudolf Baehr, Stuttgart 1998.

28 Vgl. den Kommentar in Dante Alighieri, Inferno, hrsg. v. Natalino Sapegno, Firenze 1995, S. 74. 
steht und die Verhaltensweisen der Florentiner moralisch verurteilt. Außerdem wird durch Ciaccos Prophezeiung die künftige Entwicklung von Florenz vorweggenommen, wodurch der grundsätzlichen Kritik eine zusätzliche Plausibilisierung zugeschrieben wird. Denn da es sich um eine Prophezeiung ex post handelt, wird sie durch die dem Leser zum Zeitpunkt der Lektüre bereits bekannte historische Wirklichkeit bestätigt und weckt somit den Eindruck, dass in Dantes Text empirisch überprüfbare Wahrheiten verkündet werden. Dies ist eine wichtige Selbstautorisierungsstrategie der Commedia. Sodann zeigt sich anhand der Beschreibungen und Charakterisierungen, welche Ciacco vornimmt, dass in Florenz die Wahrheit noch viel schlimmer ist, als es den Anschein hat, insofern dort nämlich Täuschung, Betrug und Habgier die leitenden Motive sind. Dies suggeriert, dass man bei der Lektüre der Commedia hinter die Kulissen blicken und ein valides Wissen über die nicht unmittelbar sichtbaren Dimensionen der Welt erwerben könne. Schließlich wird in der Begegnung mit Ciacco erstmals in der Commedia ein Gedanke formuliert, der im Folgenden immer wieder auftauchen wird, nämlich die an den Jenseitswanderer gerichtete Bitte, er möge nach seiner Rückkehr in die diesseitige Welt an den Verstorbenen erinnern: »Ma quando tu sarai nel dolce mondo, / priegoti ch'a la mente altrui mi rechi« (»Doch kommst du wieder auf die schöne Erde, / Dann bitt ich dich, von mir dort zu erzählen.«; Inf. VI, 88-89). Der Wunsch des in die Hölle verbannten Sünders nach Erinnerung in der Nachwelt steht nun aber im Gegensatz zu der durch das göttliche Strafgericht verhängten Ordnung, welche den Sünder zur Unkenntlichkeit entstellt. So sagt >Dante beim ersten Anblick des ihn ansprechenden Ciacco: "L'angoscia che tu hai / forse ti tira fuor de la mia mente, / sí che non par ch’i’ ti vedessi mai.« (»Die Angst, in der du lebest, / Hat dich vielleicht gelöscht in meinem Geiste, / So daß mir scheint, ich hab dich nie gesehen.«; Inf. VI, 43-45). Er verwendet dabei dasselbe Wort, welches Ciacco gebraucht, als er >Dante darum bittet, die noch Lebenden an ihn zu erinnern: "mente«. Durch diese Korrespondenz von ErinnertWerden und Nicht-erkennbar-Sein, welche mittels desselben Signifikanten hervorgehoben wird, manifestiert sich auf der Textoberfläche ein Widerspruch, der in vielfacher Weise in der Commedia enkodiert ist. ${ }^{29}$

29 Zur grundsätzlichen Widersprüchlichkeit von Dantes Commedia vgl. Thomas Klinkert, »Zum Verhältnis von Poesie, Politik und Metaphysik bei Dante«, in: Oliver Hidalgo/Kai Nonnenmacher (Hrsg.), Die sprachliche Formierung der politischen Moderne. Spätmittelalter und Renaissance in Italien, Wiesbaden 2015, S. 107-124. Die Gegenstrebigkeit, welche sich in der Relation zwischen Poesie, Politik und Metaphysik erkennen lässt, ist, wie ich an anderer Stelle zu zeigen versucht habe, auch auf semiotischer Ebene verankert, d. h. an die intertextuellen Verweisungsstrukturen gebunden, die einerseits Sinnkonstruktionen erzeugen und diese andererseits destabilisieren; 
Ein hier festzuhaltender, wichtiger Befund lautet daher, dass die Commedia entgegen der allgemeinen, durch maximale Ordnung gekennzeichneten Struktur des Kosmos und des diesen darstellenden Textes ${ }^{30}$ nicht frei von Spannungen und Widersprüchen ist. Diese Widersprüche sind, so meine These, das textuelle Korrelat einer Ordnung, die durch die gesellschaftliche, kulturelle und politische Entwicklung der Stadt Florenz hervorgebracht wurde. Mit anderen Worten: Florenz ist einerseits Zielscheibe von Dantes polemischen Angriffen, die sich gegen das Gewinnstreben der Kaufleute und die Zwistigkeiten der Politiker richten. Florenz ist andererseits ein »innovatives Milieu«, ${ }^{31}$ welches den Möglichkeitshorizont eines Textes wie der Commedia darstellt. In Dantes Text wird beiden Aspekten Rechnung getragen, wie im Folgenden weiter gezeigt werden soll. Dabei geht es um den Zusammenhang von Wissen, Stadt und Dichtung.

\section{Der Zusammenhang von Wissen, Stadt und Dichtung am Beispiel der Begegnung mit Farinata und Cavalcante (Inf. X)}

Die Vielschichtigkeit von Dantes Verhältnis zu Florenz manifestiert sich in besonders anschaulicher Weise im X. Gesang des Inferno. Hier kommt der Jenseitswanderer zu den Gräbern der Epikureer; Dante versteht darunter jene, die nicht an die Unsterblichkeit der Seele glauben (Inf. X, 15). Sie befinden sich im sechsten Kreis der Hölle und gehören zu den Häretikern. Im X. Gesang stehen zwei Figuren im Mittelpunkt, die auf den ersten Blick nichts miteinander zu tun haben - außer dass sie beide aus Florenz stammen: der Ghibellinenführer Farinata degli Uberti, der vor Dantes Geburt gestorben ist, und der Vater des Dichters Guido Cavalcanti, den Dante persönlich kannte. Zuerst tritt Farinata auf, der `Dante` anhand seiner Sprache als Toskaner erkennt, ohne jedoch zu wissen, um wen es sich handelt: »O Tosco che per la città del foco / vivo ten vai cosí parlando onesto, / piacciati di restare in questo loco. / La tua loquela ti fa manifesto / di quella nobil patrïa natio, / a la qual forse fui troppo molesto. (»Toskaner, der du durch die Stadt des Feuers / Lebendig schreitest und so ehrbar redest, / Du wollest hier an diesem

vgl. Thomas Klinkert, »Zum Status von Intertextualität im Mittelalter: Tristan, Lancelot, Francesca da Rimini«, in: Deutsches Dante-Jahrbuch 81 (2006), S. 27-69.

30 Die Ordnungsstrukturen des Dante'schen Textes hat Auerbach, Dante als Dichter der irdischen Welt (wie Anm. 8), herausgearbeitet.

31 Martina Heßler, »Stadt als innovatives Milieu - Ein transdisziplinärer Forschungsansatz«, in: Neue politische Literatur 47, 2 (2002), S. 193-222. 
Orte rasten. / An deiner Sprache kann man wohl erkennen, / Daß du im edlen Vaterland geboren, / Dem ich vielleicht allzu beschwerlich wurde.«; Inf. X, 22-27). Nachdem der durch die aus dem Grab sich erhebende Figur des Farinata erschrockene >Dante` von Vergil beruhigt worden ist, kommt es zu einem Zwiegespräch zwischen dem Wanderer und Farinata. Dieser, der als unerschrocken und stolz beschrieben wird, fragt >Dante nach seinen Vorfahren. Es stellt sich heraus, dass >Dantes` Familie und Farinatas Clan miteinander verfeindet sind. Farinata gehörte den Ghibellinen an, >Dante florentinischen Geschichte aufgerufen. ${ }^{32}$ Farinata war von 1239 an der Anführer der Ghibellinen und hat die Vertreibung der Guelfen im Jahr 1248 herbeigeführt. Nachdem die Guelfen 1251 in die Stadt zurückgekehrt waren, kam es erneut zu heftigen Auseinandersetzungen, die schließlich zu Farinatas Verbannung im selben Jahr führten. Der exilierte Farinata verbündete sich mit König Manfred und zog mit einem durch Truppen aus anderen toskanischen Städten verstärkten Heer gegen die Florentiner in die blutige Schlacht von Montaperti im Jahr 1260. Nach seinem Tod 1264 wurden die Ghibellinen erneut aus Florenz vertrieben, und Farinata und seine Anhänger wurden posthum wegen Häresie verurteilt. In Florenz wurde Farinata einerseits aufgrund seines rücksichtslosen Vorgehens und seines Ehrgeizes verabscheut, andererseits galt er als großer Patriot, der sich mutig den Feinden der Stadt entgegenstellte. Dass >Dante ihn eigentlich bewundert, konnten wir bei der obigen Analyse des VI. Gesanges sehen, wo er erstaunt von Ciacco erfährt, dass Farinata, den er für rechtschaffen hält, in der Hölle gelandet ist (Inf. VI, 77-87).

Im X. Gesang erscheint Farinata als eine furchteinflößende Figur und als einer, der offenbar keine Angst vor den Qualen der Hölle hat (vgl. V. 36, »com'avesse l'inferno a gran dispitto« [»Als wollte er die Hölle tief verachten«]). Als er erfährt, dass >Dantes` Vorfahren seine Feinde waren, zieht er - wie man vermuten kann, leicht indigniert - die Augenbrauen hoch und teilt kühl mit, dass er diese seine Gegner zweimal aus der Stadt vertrieben habe; worauf >Dante، entgegnet, dass die Guelfen nach der Vertreibung jeweils wieder zurückgekommen seien, wohingegen die Ghibellinen die Kunst der Rückkehr nicht so gut beherrscht hätten. Schon an diesem Wortwechsel zeigt sich die Ambivalenz von >Dantes` Einstellung Farinata gegenüber. Einerseits ist er von ihm eingeschüchtert, andererseits lässt er sich seine herablassenden Worte nicht gefallen und kontert durchaus geistreich und schlagfertig.

32 Vgl. die Erläuterungen im Kommentar von Sapegno, Inferno (wie Anm. 28), S. 114-115, auf die ich hier zurückgreife. 
An dieser Stelle wird das Gespräch mit Farinata jedoch unterbrochen, indem unvermittelt ein anderer Höllenbewohner auftaucht, nämlich Cavalcante, der Vater des Dichters Guido Cavalcanti. Dieser scheint sofort zu wissen, wer >Dante ist, denn er erkundigt sich nach seinem Sohn, dessen Freundschaft mit dem Wanderer ihm bekannt ist. Offenbar kennen sich auch >Dante und Cavalcante, denn anders als bei Farinata muss der Jenseitswanderer nicht erst fragen, um wen es sich handelt, und auch umgekehrt muss Cavalcante nicht darüber aufgeklärt werden, wen er vor sich hat. Im Nachhinein erklärt der Erzähler, woran er Cavalcante erkannt habe, nämlich zum einen an seiner Rede (»Le sue parole«, Inf. X, 64), zum anderen an der Art seiner Strafe. Denn auch er gehört zu den Epikureern, die nicht an ein Weiterleben der Seele nach dem Tod glauben. Dies ergibt sich auch aus der Frage, die er >Dante`stellt. Er vermutet nämlich, dieser sei aus eigener Initiative in die Hölle gekommen (»per altezza d'ingegno«, V. 59). >Dante k klärt ihn jedoch darüber auf, dass er nicht allein gekommen sei, sondern begleitet von einer höheren Instanz, nämlich Vergil, der, wie der Leser aus Inf. II, 94 ff. weiß, im Auftrag Beatrices und der göttlichen Macht handelt. Folgendermaßen lautet der Wortlaut von >Dantes` Anrede an Cavalcanti: " DDa me stesso non vegno: / colui ch'attende là, per qui mi mena / forse cui Guido vostro ebbe a disdegno«" (»IIch komme nicht alleine. / Der, der dort wartet, hat mich hergeleitet. / Vielleicht hat Euer Guido ihn verachtet.«; Inf. X, 61-63). Diese Stelle gehört zu den meistkommentierten der Commedia; ihr Sinn ist schwer zu erschließen. ${ }^{33}$ Eine Möglichkeit, die Stelle $\mathrm{zu}$ begreifen, ist folgende: "colui ch'attende là» bezieht sich auf Vergil, der >Dante < begleitet und sich in dieser Szene abseits hält. Er ist derjenige, der >Dante « »per qui«, »hierher«, d. h. in den Raum der göttlichen Wahrheit führt. Der Relativsatz »cui Guido vostro ebbe a disdegno« wäre zu beziehen auf den Sachverhalt, dass >Dante` von Vergil im göttlichen Auftrag in den Bereich der göttlichen Wahrheit geführt wird, einen Sachverhalt, mit dem sich Guido Cavalcanti offenbar nicht anfreunden wollte (»ebbe a disdegno«), vielleicht weil er wie Farinata und sein eigener Vater ein Epikureer ist. Was die Stelle darüber hinaus bedeuten kann, was hier indes nur angedeutet wird, ist durch intertextuelle Verweisungsstrukturen zu rekonstruieren.

Bevor ich zu diesen Verweisungsstrukturen komme, soll die vorliegende Textstelle noch etwas weiter analysiert werden. Cavalcantis Vater richtet sein Interesse ausschließlich auf seinen Sohn. Aus `Dantes im Vergangenheitstempus stehender Aussage über Guido Cavalcanti (»ebbe a disdegno«) schließt dessen Vater zu

$33 \mathrm{Zu}$ den unterschiedlichen syntaktischen Lesarten der Stelle vgl. Mario Marti, „Cavalcanti, Guido«, in: Umberto Bosco (Hrsg.), Enciclopedia dantesca, Bd. I, Roma 1970, S. 891-896, hier S. 895. 
Unrecht, dass Guido schon gestorben sei. ${ }^{34}$ Bevor >Danteく dieses Missverständnis aufklären kann, verschwindet der Schattenkörper des Vaters wieder in seinem Grab. Nach dieser Unterbrechung setzt `Dante`seinen Dialog mit Farinata fort, der an der Stelle wieder aufgegriffen wird, an der er abrupt unterbrochen worden war. Farinata greift das Stichwort »arte« (Inf. X, 51, 77) auf - gemeint ist die Kunst, nach einer Verbannung wieder in die Stadt zurückzukehren - und bedauert, dass seine Ghibellinenpartei diese Kunst weniger gut beherrscht habe als die der gegnerischen Guelfen. Seinerseits rächt er sich nun aber an `Dante`, indem er ihm selbst das Exil prophezeit. Sodann fragt er `Dante`, warum man in Florenz seine Partei so hart bestrafe, was `Dante` mit dem Hinweis auf die Schlacht bei Montaperti erklärt. Farinata verteidigt sich, indem er darauf hinweist, dass er für diese Schlacht nicht der einzige Verantwortliche gewesen sei, und indem er zu seinen Gunsten anführt, dass er allein Florenz verteidigt habe, als alle anderen es zugelassen hätten, dass es von seinen Feinden vernichtet worden wäre. Man merkt an dem Dialog, den `Dante mit Farinata führt, dass die beiden sich nichts schenken. Sie setzen den erbitterten Kampf ihrer politischen Gruppierungen mit Worten fort.

Im letzten Teil der Begegnung mit Farinata erhält >Dante von ihm eine Erklärung für die in seinen Augen seltsame Tatsache, dass die Verstorbenen zwar über die Zukunft Bescheid wissen, nicht aber über die Gegenwart. Dieses Rätsel ergibt sich für >Dante` aus der Diskrepanz zwischen Farinatas Prophezeiung und Cavalcantes Nichtwissen. Die beiden Figuren, die zunächst scheinbar überhaupt nichts miteinander zu tun haben, rücken jetzt in einen Sinnzusammenhang. Sie verkörpern offenbar paradigmatisch zwei typische Eigenschaften von Jenseitsbewohnern, welche es zu erklären gilt. Es geht dabei um die Diskrepanz zwischen Wissen und Nichtwissen, von der ja auch schon oben im Zusammenhang mit Inf. VI die Rede war. Farinata erklärt diese Diskrepanz mit einer medizinischen Analogie. So wie ein Weitsichtiger nur in der Ferne klar sehe, in der Nähe aber die Welt undeutlich wahrnehme, könnten die Jenseitsbewohner nur die in der Ferne liegende Zukunft, nicht aber die nahe Gegenwart wahrnehmen. Dies bringt >Dante $\mathrm{zu}$ der retrospektiven Erkenntnis, dass er sich gegenüber Cavalcantis Vater falsch verhalten habe. Er habe, so gibt er zur Begründung an, Cavalcante deshalb nicht auf seine Frage nach dem Sohn geantwortet, weil er über diesen Widerspruch zwischen Wissen und Nichtwissen nachgedacht habe, und er bittet Farinata, dies dem Vater seines Freundes mitzuteilen.

Versuchen wir die einzelnen Komponenten des analysierten Canto abstrakter zu benennen, so können wir sagen, dass es bei der Begegnung mit den Epikureern

34 Der Dichter Guido Cavalcanti starb im August 1300, während die in der Commedia erzählte Jenseitsreise der textinternen Chronologie zufolge an den Ostertagen desselben Jahres stattfindet. 
um Folgendes geht: (1) die Stadt Florenz und ihre Geschichte, (2) den Gegensatz zwischen Wissen und Nichtwissen, zwischen Blindheit und Erkenntnis, (3) die persönliche Geschichte Dantes, die eng mit der Geschichte seiner Heimatstadt verknüpft ist. Farinata ist Teil der Geschichte von Florenz vor Dantes Geburt. Cavalcante ist der Vater eines Freundes und Dichterkollegen von Dante. Sowohl Farinata als auch Cavalcante gehören derselben Florentiner Partei an, und Dante und sein Dichterkollege Cavalcanti sind Teil einer Dichterbewegung, die man heute als den »(dolce) stil nuovo « bezeichnet. ${ }^{35}$ All dies steht so nicht explizit im Text. Die heutigen Leser wissen es aber dank der Kommentarliteratur und dank der literarischen Kanonisierung. Ein Leser des frühen 14. Jahrhunderts, der noch auf keinerlei Kommentare zurückgreifen kann, kann dies alles nur verstehen, wenn er über Insiderwissen aus der Stadt Florenz verfügt. Daraus lässt sich schlussfolgern, dass der Text sich an einen solchen idealen Leser mit Insiderwissen über Florenz wendet. Und genau dies kommt in dem zu Beginn dieses Beitrags analysierten Bild von Michelino zum Ausdruck, welches die Commedia als einen Kommunikationsakt zwischen Dante und seiner Heimatstadt inszeniert.

Es ist nun noch genauer über Guido Cavalcanti nachzudenken. Dieser Dichterkollege und Freund spielt eine wichtige Rolle für Dantes eigenen dichterischen Werdegang. Vor dem entscheidenden Wendepunkt der Vita nova, welcher durch das Gedicht »Donne ch'avete intelletto d'amore« markiert wird, befindet sich ein Gedicht, welches von vielen Interpreten als »cavalcantianisch« aufgefasst wird und welches folgendes Incipit hat: »Spesse fiate vegnon mi alla mente». In diesem Gedicht ist von den »oscure qualità « Amors die Rede, den lebensbedrohlichen Qualen der Liebe, der Unentrinnbarkeit des Liebesleids. Diese Schmerzen, welche aus einer unerfüllbaren Liebe resultieren, versucht Dante sodann durch eine neue Haltung zu überwinden, die darauf abzielt, die Geliebte nicht mehr physisch zu begehren, sondern sie rein spirituell zu lieben und zu loben. In dieser »svolta« sieht Dante seine eigentliche Leistung als Liebeslyriker, seine Überwindung der Tradition, und damit aller Vorläufer bis hin zu den engsten Freunden,

35 Mario Marti, „Stil nuovo«, in: Umberto Bosco (Hrsg.), Enciclopedia dantesca, Bd. V, Roma 1976, S. 438-444, hier S. 438-439: „Stil nuovo. - Con questa formula scolastica la moderna storiografia della letteratura italiana significa i temi e i modi espressivi di un gruppo di giovani poeti toscani vissuti a cavallo dei secoli XIII e XIV, i quali, esplicitamente in loro dichiarazioni o implicitamente con la loro stessa attività letteraria, riconobbero come loro anticipatore il bolognese Guido Guinizzelli. Essi sono: Guido Cavalcanti, Cino da Pistoia, Lapo Gianni, Gianni Alfani, Dino Frescobaldi e D[ante] in un preciso periodo della sua attività giovanile. La formula di ১Stil nuovor, per quanto sia entrata nell'uso e tenda a diffondersi ulteriormente forse per la sua maggiore ampiezza connotativa o forse soltanto per la sua più comoda rapidità, deriva dall'altra di `Dolce stil nuovor, che è esatta e piena definizione dantesca appunto utilizzata come etichetta storiografica dell’attività letteraria di quel gruppo e del suo anticipatore.» 
deren wichtigster Guido Cavalcanti war. ${ }^{36}$ In Purg. XXIV inszeniert er sich selbst in entsprechender Weise: Der Lyriker Bonagiunta da Lucca spricht `Dante ‘ auf die von ihm hervorgebrachte, neuartige Lyrik an, indem er auf das diese Lyrik einleitende Gedicht verweist, »Donne ch'avete intelletto d'amore«, worauf >Dante` ihm antwortet, er tue nichts anderes, als das aufzuschreiben, was Amor ihm diktiere (Purg. XXIV, 49-54). Bonagiunta stimmt ihm mit folgenden Worten zu:

»O frate, issa vegg'io«, diss'elli, »il nodo

che 'l Notaro e Guittone e me ritenne

di qua dal dolce stil novo ch'i' odo!

Io veggio ben come le vostre penne

di retro al dittator sen vanno strette,

che de le nostre certo non avvenne;

e qual piú a gradire oltre si mette,

non vede piú da l'uno a l'altro stilo«;

e, quasi contentato, si tacette. (Purg. XXIV, 55-63)

»O Bruder«, sprach er, »nun seh ich den Knoten,

Der den Notar, Guitton und mich noch ferne

Von jenem süßen neuen Stil gehalten.

Ich sehe wohl, wie folgsam eure Federn,

Dem, der diktiert, auch auf sein Wort gehorchen;

Das ist mit unsern freilich nicht geschehen.

$36 \mathrm{Zu}$ Dantes Verhältnis zu Cavalcanti vgl. u. a. Marti, »Cavalcanti, Guido« (wie Anm. 33), Gianfranco Contini, »Cavalcanti in Dante«, in: ders., Un'idea di Dante. Saggi danteschi, Torino 1976, S. 143-157, Nievo Del Sal, »Cavalcanti in Dante >comico«", in: Rivista di letteratura italiana 9 (1991), S. 9-52, sowie Teodolinda Barolini, »Dante and Cavalcanti (On Making Distinctions in Matters of Love): Inferno 5 and Its Lyric and Autobiographical Context «, in: dies., Dante and the Origins of Italian Literary Culture, New York 2006, S. 70-101. Barolini bringt den Unterschied zwischen den Liebeskonzeptionen Cavalcantis und Dantes als des Autors der Commedia auf folgenden prägnanten Nenner: „What Dante considers, in fact, to be the very definition of lust rather than love - carnal sinners are defined precisely as those who subject reason to desire Cavalcanti considers to be the very nature of love: all love. So, while we are accustomed to thinking of Inferno 5 as a text that forces us to deconstruct Francesca's use of the word amore [...] we can now see that it is also a text that protects and defends love (what Dante would classify as real love) from a blanket indictment like that of Cavalcanti.« (S. 81-82) Barolini weist nach, dass in Inf. $\mathrm{V}$ ein unterschwelliger Dialog mit Cavalcanti geführt wird. Dieser implizite Dialog mit Cavalcanti betreffe die Commedia insgesamt; Barolini spricht von »our persistent sense of a pervasive Cavalcantian presence in the Commedia that goes far beyond explicit citations of or referrals to Guido« (S. 79). Diese unterschwellige Präsenz Cavalcantis wird in dem im vorliegenden Beitrag untersuchten Canto (Inf. X) auf raffinierte Weise inszeniert. 
Und wer noch weiter sich vertiefen möchte,

61

Wird nichts mehr finden zwischen beiden Stilen.«

Und damit schwieg er und war fast zufrieden.

In dieser berühmten Stelle wird die von den Philologen des 19. Jahrhunderts zum literarhistorischen Fachbegriff erhobene Prägung »dolce stil novo« erstmals verwendet, und zwar als eine Stilqualität, die Dante und die ihm nahestehenden Lyriker - Bonagiunta sagt »le vostre penne« und meint damit die Federn von Dante und seinen Dichterfreunden - von den Vorläufern unterscheidet. Von entscheidender Bedeutung ist hier das Lexem »il nodo«, was wörtlich »der Knoten«, im übertragenen Sinne »das Hindernis« bedeutet. $^{37}$ Dieses Lexem wird hier als Schlüsselbegriff einer Differenz verwendet, nämlich der Differenz zweier Arten zu dichten, und es wird verbunden mit einer retrospektiven Einsicht, einer Erkenntnis, die durch das Verbum "vedere« angezeigt wird, welches im Text gleich zweimal vorkommt: »issa vegg'io« (Purg. XXIV, 55) und »Io veggio ben« (V. 58).

Die Lexemverbindung von »nodo« und »vedere« kommt nun aber auch in Inf. $\mathrm{X}$ vor, jenem Canto, in dem >Dante Wissens der Jenseitsbewohner erhält. >Dante< bittet ihn: »solvetemi quel nodo / che qui ha 'nviluppata mia sentenza." (»dann löset mir den Knoten, / Der hier mein Denken in sich hat verschlungen«; Inf. X, 95-96). Hier bedeutet "nodo« soviel wie >Hindernis des Verstehens` oder >Blockade des Denkens` und bezieht sich auf die oben bereits analysierte Diskrepanz zwischen zukunftsbezogenem Wissen und gegenwartsbezogenem Nichtwissen. Zweimal kommt auch hier das Verbum "vedere« vor (Inf. X, 97 und 100). Entscheidend ist nun, dass dieser Teil des Canto zurückverweist auf die abrupt beendete Begegnung mit Cavalcantis Vater. Der »nodo«, der `Dantes« Verständnis blockiert hatte und hier von Farinata aufgelöst wird, war die Ursache für die nichtgegebene Antwort `Dantes` in Bezug auf Guido Cavalcanti. Dieser Dichterfreund, nach dem der Vater ja gefragt hatte, wurde von `Dante an jener Stelle verschwiegen. Er ist, so kann man das ausdeuten, seiner Denkblockade zum Opfer gefallen.

Diese Leerstelle ist ein entscheidendes bedeutungstragendes Element in Inf. X. Sie verbirgt nämlich jene Dimension, die, neben der Erkenntnisproblematik und der Bedeutung der Stadt Florenz, für die Konzeption der Commedia ebenfalls maßgeblich ist: die Dichtung. Dante ist ein selbstbewusster Autor, der einen hohen Innovationsanspruch für seine Dichtung erhebt und diesen Anspruch auch in seinem Text deutlich artikuliert. Dass er den Anspruch in diesem Canto al-

37 Vgl. hierzu ausführlich Alice Malzacher, »Il nodo che ... me ritenne«. Riflessi intertestuali della Vita Nuova di Dante nei Rerum vulgarium fragmenta di Petrarca, Firenze 2013. 
lerdings nur indirekt markiert, ist ein Zeichen für das implizite Wissen, welches der Text in Bezug auf seine eigene Genese enthält. Es geht Dante darum, sich von bestimmten Formen der Dichtung, die im Florentiner Milieu des späten 13. Jahrhunderts durch Begegnungen mit anderen Dichtern in seinem Horizont lagen, zu distanzieren und diese zu überwinden. Er tut dies, indem er einen Autor wie Cavalcanti, dem er Vieles zu verdanken hat, in der beschriebenen Art und Weise sichtbar unsichtbar macht.

\section{Schluss}

Es war Anliegen dieses Beitrags, den Zusammenhang von Urbanität und Literatur am Beispiel Dantes zu untersuchen. Ausgangspunkt war das Fresko des Malers Domenico di Michelino, welches die Divina Commedia als einen Kommunikationsakt inszeniert, dessen privilegierter Adressat die Stadt Florenz ist. Mit dieser Deutung trifft Michelino den Kern des Dante'schen Werks. Wie die Analyse zweier Canti, des VI. und des X. Gesanges des Inferno, ergeben hat, lässt sich die Commedia als das textuelle Korrelat einer Wissensordnung begreifen, die durch die gesellschaftliche, kulturelle und auch politische Entwicklung der Stadt Florenz im 13. und frühen 14. Jahrhundert ermöglicht wurde. Der sich in der Struktur der Commedia in mehrfacher Weise artikulierende Grundwiderspruch besteht darin, dass Dante einerseits eine radikale Kritik an der Händlermentalität und der politischen Zerrissenheit seiner Heimatstadt übt, und andererseits genau weiß, was er dem innovativen Milieu dieser Stadt als Dichter und philosophischer Denker zu verdanken hat. Außerdem konnte anhand der Begegnung mit den Epikureern Farinata und Cavalcante in Inf. X gezeigt werden, dass diese Textstellen nur von einem Leser mit Insiderwissen über die Stadt Florenz, ihre Geschichte, ihre Parteien und vor allem auch ihre Dichterkreise adäquat rezipiert werden können. Mindestens ebenso wichtig wie das Gesagte ist das von Dante verschwiegene Wissen, welches er bei seinem Leser voraussetzt und welches die Kommentarliteratur seit dem 14. Jahrhundert dem Text hinzugefügt hat. Jenseits der rein thematischen Dimension ist das Urbane somit Teil der Textstruktur und des Kommunikationsgeschehens der Commedia. Die Stadt wird von Dante seinem Text >eingeschrieben`.

\section{Abbildungsnachweis}

Abb. 1: Domenico di Michelino, Die Allegorie der Göttlichen Komödie (1465). Fresko, Santa Maria del Fiore, Florenz. (C) Gemeinfrei. 